\title{
A random walker on a ratchet
}

\author{
José L. Mateos ${ }^{1}$ \\ Instituto de Física, Universidad Nacional Autónoma de México, \\ Apartado Postal 20-364, 01000 México, D.F., México
}

\begin{abstract}
We analyze a model for a walker moving on a ratchet potential. This model is motivated by the properties of transport of motor proteins, like kinesin and myosin. The walker consists of two feet represented as two particles coupled nonlinearly through a bistable potential. In contrast to linear coupling, the bistable potential admits a richer dynamics where the ordering of the particles can alternate during the walking. The transitions between the two stable states on the bistable potential correspond to a walking with alternating particles. We distinguish between two main walking styles: alternating and no alternating, resembling the hand-over-hand and the inchworm walking in motor proteins, respectively. When the equilibrium distance between the two particles divided by the periodicity of the ratchet is an integer, we obtain a maximum for the current, indicating optimal transport.
\end{abstract}

Key words: Noise; Transport; Brownian motors; Ratchets

PACS: 05.40.-a; 02.50.Ey; 05.60.Cd; 05.10.Gg

\section{Introduction}

In recent years, advances in non-equilibrium statistical physics have revealed various instances of the surprising phenomenon of noise enhanced order, such as stochastic resonance $[1,2,3]$, Brownian motors, ratchets or noise-induced transport $[4,5,6]$. These remarkable phenomena occur due to the constructive role of noise in nonlinear dynamical systems [7]. Noise-induced, directed transport in a spatially periodic system in thermal equilibrium is ruled out by the second law of thermodynamics. Therefore, in order to generate transport, the

1 E-mail: mateos@fisica.unam.mx;

Fax: (52) (55) 5622 5015; Phone: (52) (55) 56225130

Preprint submitted to Elsevier Science 21 November 2018 
system has to be driven away from thermal equilibrium by an additional deterministic or stochastic force. In the most interesting situation, these forces are unbiased, that is, their temporal, spatial or ensemble averages vanish. Besides the breaking of thermal equilibrium, another important requirement to get directed transport in a spatially periodic system is the breaking of the spatial inversion symmetry. We speak then of Brownian motors, ratchet potentials, or, in the biological realm, of molecular motors. This recent burst of work is motivated in part by the challenge to explain the unidirectional transport of molecular motors in the biological realm $[8,9,10]$.

One particular motor protein, kinesin, has attracted considerable attention, motivated by experimental results in which the dynamical details of its motion can be measured $[8,10]$. Kinesin is a protein with two heads that perform a walking on microtubules inside cells. Motivated by these experimental results, several authors $[11,12,13,14,15,16,17,18]$ have introduced diverse models in order to understand the particular walking of kinesin. Usually, these models consider two coupled particles on a ratchet potential that represents the periodic asymmetric structure of microtubules. In these papers the authors consider a linear elastic coupling between the particles. This coupling implies that the order of the particles cannot change. However, in recent experiments, it was found that kinesin moves processively alternating its two heads in a way called hand over hand $[19,20,21]$. Other processive motor proteins that move on actin filaments in a hand-over-hand way are myosin $\mathrm{V}[22,23]$ and myosin VI $[24,25]$.

We introduced in this paper a model inspired by the walking of motor proteins, like kinesin on microtubules, but that is not restricted to walking of motor proteins. The model can be useful to describe as well the walking of macroscopic objects in the presence of fluctuations. It consists of two particles coupled through a nonlinear bistable potential and subjected to independent white noises. This system of two coupled particles is acted upon by a spatiallyperiodic force, due to the presence of a ratchet potential and, additionally, we have a common time-dependent periodic force. We are interested in analyzing the trayectories of the walker following in detail the motion of each of the particles, and we are also interested in the current or noise-induced transport for this system.

\section{The model of a walker with two Brownian motors}

The model considers a walker moving on an asymmetric ratchet potential mediated by noise. This walker has two feet that are represented as two particles coupled nonlinearly through a bistable potential $[26,27,28]$. The walker moves along a track formed by an asymmetric ratchet potential, and is subjected to 
the influence of two independent white noises acting on the two particles and a common external harmonic force. The stochastic differential equations for the two particles, represented by $x$ and $y$, in the overdamped regime, are:

$$
\begin{aligned}
& m \gamma \dot{x}=-\partial_{x} V(x)-\partial_{x} V_{b}(x-y)+m \gamma \sqrt{2 D} \xi_{1}(t)+F_{D} \sin (\Omega t+\varphi) \\
& m \gamma \dot{y}=-\partial_{y} V(y)-\partial_{y} V_{b}(x-y)+m \gamma \sqrt{2 D} \xi_{2}(t)+F_{D} \sin (\Omega t+\varphi) .
\end{aligned}
$$

where $m$ is the mass of each particle, $\gamma$ is the friction coefficient, $-\partial_{x} V(x)$ is the force due to the ratchet potential, $-\partial_{x} V_{b}(x-y)$ is the coupling force due to the bistable potential. The common external harmonic force has three parameters: the amplitude force $F_{D}$, the frequency $\Omega$ and the initial phase $\varphi$.

These equations represent two coupled particles on a periodic asymmetric ratchet potential given by [29]

$$
V(x)=V_{1}-V_{R}\left[\sin \frac{2 \pi\left(x-x_{0}\right)}{L}-\frac{1}{4} \sin \frac{4 \pi\left(x-x_{0}\right)}{L}\right] .
$$

where $L$ is the period of the potential $V(x+L)=V(x)$; the other constants will be discussed later. Additionally, these particles are coupled by the nonlinear cubic force coming from a bistable potential $V_{b}(x-y)$ given by

$$
V_{b}(x-y)=V_{b}+V_{b}\left[\frac{(x-y)^{4}}{l^{4}}-2 \frac{(x-y)^{2}}{l^{2}}\right] .
$$

Here, $V_{b}$ is the amplitude of the bistable potential and represents the coupling strenght between the particles, and $2 l$ is the distance between the two minima.

Finally, the parameter $D$ is the intensity of the zero-mean statistically independent Gaussian white noises $\xi_{1}(t)$ and $\xi_{2}(t)$ acting on particles $x$ and $y$, respectively. Being statistically independent, the following equation is satisfied:

$$
\left\langle\xi_{i}(t) \xi_{j}(s)\right\rangle=\delta_{i j} \delta(t-s)
$$

Let us derive now dimensionless equations of motion for the model. We use as the characteristic lenght scale the period of the ratchet potential $L$, the characteristic time scale will be given by the inverse of the friction coeficient $\tau=1 / \gamma$, and the charactersitic force is $m L \gamma^{2}$. Let us define the following dimensionless units: $x^{\prime}=x / L, x_{0}^{\prime}=x_{0} / L, y^{\prime}=y / L, y_{0}^{\prime}=y_{0} / L, t^{\prime}=\gamma t$, $l^{\prime}=l / L, \Omega^{\prime}=\Omega / \gamma$ and $D^{\prime}=D / \gamma L^{2}$. The dimensionless equations of motion, after renaming the variables again without the primes, become 


$$
\begin{aligned}
& \dot{x}=-\partial_{x} V(x)-\partial_{x} V_{b}(x-y)+\sqrt{2 D} \xi_{1}(t)+A \sin (\Omega t+\varphi), \\
& \dot{y}=-\partial_{y} V(y)-\partial_{y} V_{b}(x-y)+\sqrt{2 D} \xi_{2}(t)+A \sin (\Omega t+\varphi),
\end{aligned}
$$

The dimensionless ratchet potential is

$$
V(x)=C-U_{R}\left[\sin 2 \pi\left(x-x_{0}\right)+\frac{1}{4} \sin 4 \pi\left(x-x_{0}\right)\right] .
$$

The constant $C=-U_{R}\left(\sin 2 \pi x_{0}+0.25 \sin 4 \pi x_{0}\right)$ is such that $V(0)=0$. The constant $x_{0}$ is introduced in order to center the minima of the periodic potential on the integers [29].

The dimensionless bistable potential is given by

$$
V_{b}(x-y)=U_{b}\left[1+\frac{(x-y)^{4}}{l^{4}}-2 \frac{(x-y)^{2}}{l^{2}}\right]
$$

Here the dimensionless amplitude of the ratchet potential is given by $U_{R}=$ $V_{R} /\left(m L^{2} \gamma^{2}\right)$, and the dimensionless amplitude of the bistable potential $U_{b}=$ $V_{b} /\left(m L^{2} \gamma^{2}\right)$. The amplitude of the external force is $A=F_{D} /\left(m L \gamma^{2}\right)$.

In Fig. 1 we depict the ratchet (solid line) and the bistable (dashed line) potential for this model. The dotted line is the sum of both potentials. As is clear in the figure, the total potential has three minima instead of two, due to the interplay between the stable points in both potentials. These means that we have three stable equilibria configurations for the walker: $-l, 0$ and $l$. In our model the situation is even more complicated since we have two particles in a potential like in Fig. 1. We can think of this problem as a single particle in a two-dimensional potential given by $\Psi(x, y)=V(x)+V(y)+V_{b}(x-y)$. A discussion of this $2 \mathrm{D}$ problem and the analysis of its bifurcations can be seen in $[26,27,28]$.

This model is different from previous ones described in the literature, since it incorporates a nonlinear coupling between the two particles through the bistable potential, as has been discussed before $[26,27,28]$. It is important to stress the following: the coupling through the bistable potential involves the variable $x-y$. This variable can be positive, negative or zero. When $x-y>0$ the $x$ particle is ahead of the $y$ particle. On the other hand, when $x-y<0$ the $y$ particle is ahead of the $x$ particle. Therefore, the transitions between the two stable states in the bistable potential correspond to an exchange of the order between the particles. The minima, corresponding to the two stable points in the bistable potential, are located at $x-y=l$ and $x-y=-l$, that is, when the distance between the two particles is $l$. Thus, we have two 


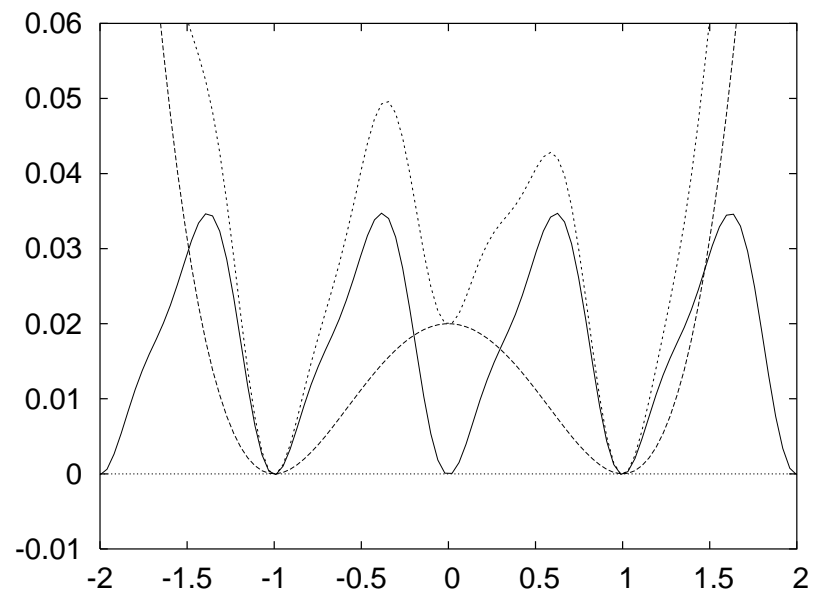

Fig. 1. The ratchet (solid line) and bistable potential (dashed line) for the random walker model in the case $l=1$. The dotted line is the sum of both potentials.

equilibrium configurations for the walker: $x-y=l$ and $x>y$, or $x-y=-l$ and $x<y$. The local maximum at the origin in the bistable potential is unstable without the ratchet, but it can become a third stable configuration in the presence of the ratchet potential, as can be seen in Fig. 1. This stable state corresponds to the case when $x-y=0$, that is, when the two particles coincide in space. So, we can think of a state oscillating in the bistable potential back and forth between the two minima, as the walking of a motor protein alternating its two heads, or a walker alternating its two feet. This nonlinear coupling allow us then to consider a very important aspect of a real walking that was lacking in previous models: the possibility of alternating the two feet. In the models given in $[11,12,13,14,17,18]$, the coupling between the particles is linear (a harmonic spring) and thus the particles cannot alternate positions. They simply can approach to each other, but once you have an ordering, say $x>y$, the ordering remains for the rest of the dynamics. In our case, on the other hand, we can have several types of walking: alternating random walking, where the two particles alternate their order randomly when the walker moves through the ratchet (hand over hand) or; rigid random walking, where the two particles move on the ratchet without exchanging their order (inchworm).

Coming back to the stochastic equations that define our model, we notice that an important parameter is $r=U_{b} / U_{R}$, the ratio of two barrier heights: the bistable and the ratchet. Both are energetic barriers that have to be overcome in order to perform a particular walking: alternating or rigid. If we overcome only the ratchet barrier, then we have a rigid walking, whereas if the walker can overcome both barriers, then the walking can alternate the feet. From this particular coupling we see a clear connection between the phenomena of stochastic resonance and Brownian motors. One expects that in an optimal situation, aided by the stochastic resonance mechanism, one can transit very efficiently between the two states in the bistable potential and at the same 


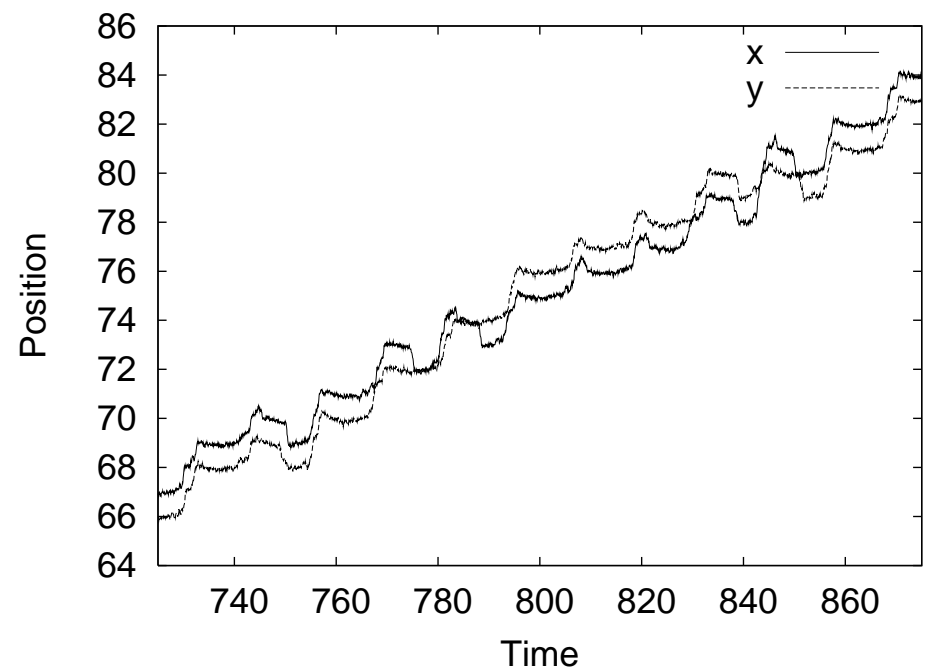

Fig. 2. A typical trajectory of a walker moving along the ratchet. The solid line represents the $x$ particle and the dashed line the $y$ particle. The parameters are: $A=1.0, D=0.1, \Omega=0.5, l=1.0$ and $r=0.78$.

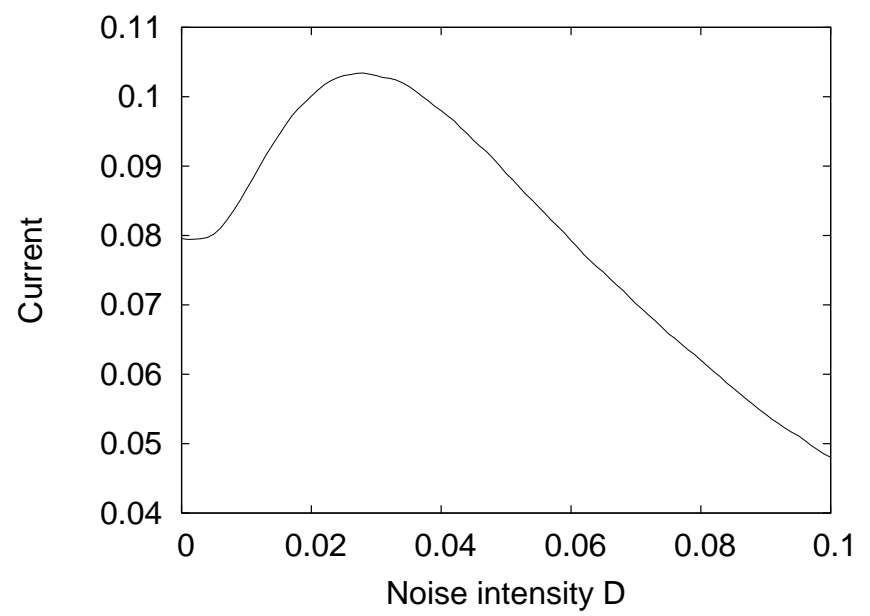

Fig. 3. The current as a function of noise intensity $D$. The parameters are: $A=1.0$, $\Omega=0.5, l=1.0$ and $r=1.57$.

time walk optimally on the ratchet potential using an alteration of the two particles. This might happen when the equilibrium distance $l$ between particles coincide with the periodicity of the ratchet potential. In our units this case corresponds to $l=1$.

\section{Numerical results}

In this section we will solve numerically the Langevin equations of motion to obtain the trajectories of the walker. We use a stochastic fourth-order Runge- 


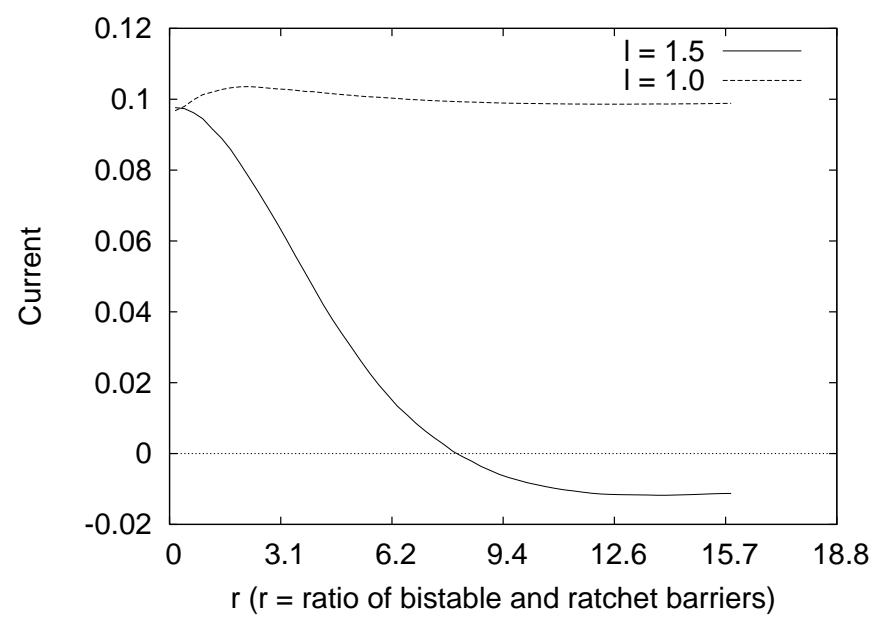

Fig. 4. The current as a function of the ratio of the amplitudes of the ratchet and bistable potential barriers $r$. The dashed line corresponds to $l=1$ and the solid line to $l=1.5$. Notice the current reversal for the latter case. The parameters are: $A=1.0, D=0.03$ and $\Omega=0.5$.

Kutta algorithm to solve the system of stochastic differential equations with additive Gaussian white noise. This means that we use a fourth-order RungeKutta algorithm for the deterministic part and a random number generator to obtain Gaussian-distributed random numbers from uniformly distributed ones in the unit interval, using the Box-Mueller algorithm, as described in [30]. We calculate the current, which is simply the ensemble average velocity of the center of mass of the walker, where the center of mass is given by $z(t)=(x(t)+y(t)) / 2$. In the numerical results that we present now, we fix the value of $\Omega=0.5$. The other parameters are indicated in the text or in the figure captions. The quantities that we compute involve an averaging of an ensemble with different initial random phases $\varphi$ uniformly distributed on a circle between 0 and $2 \pi$.

In Fig. 2 we show a typical trajectory for the walker. The solid line corresponds to the $x$ particle and the dashed line to the $y$ particle. Notice that due to the coupling the two particles tend to move together and the center of mass advance with a positive current. As can be seen in the figure, the two feet can exchange positions and different types of walking patterns can be observed. For instance, the walker can overcome the ratchet barriers without exchanging the order of the particles for a while, but later on the feet tend to be together and the walker jumps in this way to the next minimum in the ratchet and finally the order of the feet can change. In Fig 3 we show the current as a function of noise intensity. We notice that the current increases until it reaches a maximum and then tend to decrease. This remind us the phenomenon of stochastic resonance. For zero noise the current is finite, since we have a large amplitude $A=1$ for the external forcing. So we obtain a nonzero current even in the deterministic limit. In Fig. 4 we depict the current as a function 


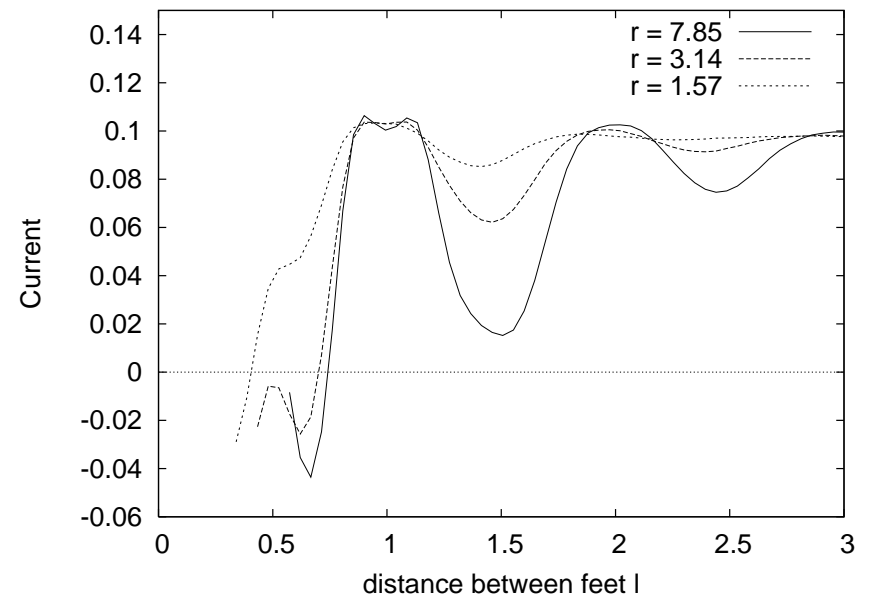

Fig. 5. The current as a function of the equilibrium distance between particles $l$. The different curves correspond to different values of the ratio $r$. The parameters are: $A=1.0, D=0.03$ and $\Omega=0.5$.

of $r$, the ratio between the bistable and ratchet potentials, for two values of the equilibrium distance between feet $l$. For $l=1$ we see that the current is almost constant, but for $l=1.5$ the current depends strongly on $r$ and can even reverse sign. So we obtain a current reversal as a function of the ratio $r$. Finally, in Fig. 5 we show the current as a function of the equilibrium distance between feet $l$, for different values of the ratio $r$. We did not show the current for small values of $l$, since in this case the bistable potential can be very large in comparison with the other terms in the Langevin equations. We notice that the current attains a maximum when $l$ is near an integer. In some case the maximum is not exactly at the integers, but close to it. In fact, for large values of the ratio $r$ (for instance $r=7.85$ in Fig. 5), the current develops a local minimum at $l=1$. We are planning to analyze further this effect for other parameters in order to determine its possible generality. This means that the walker can move through the ratchet in a very efficient way when each particle is located close to a minimum of the ratchet. On the other hand, if the distance $l$ is in between integers, the current attains a minimum, indicating that the walker is unable to move efficiently.

\section{Concluding remarks}

In summary, we have introduced a model for a random walker that consists of two particles coupled nonlinearly through a bistable potential, steeping on an asymmetric periodic ratchet potential. In contrast to linear coupling, the bistable potential admits a richer dynamics where the ordering of the particles can alternate. The dynamics then include two typical stepping patterns: alternating (hand over hand) and non alternating walking (inchworm). In our 
model we can obtain both types of walking, depending on the ratio between the ratchet and the bistable barriers, and on the ratio between the equilibrium distance of the particles and the periodicity of the ratchet. It is worth mentioning that according to recent experiments in motor proteins $[19,20,21,22,23,24,25]$ the hand over hand type of walking is more likely. We have calculated the current, defined as the average velocity of the center of mass, as a function of parameters related with the coupling and the distance between particles. In the case where the equilibrium distance between particles is a multiple of the periodicity of the ratchet potential, we obtained a maximum value for the current. Thus, we have a new model that may shed light on a number of currently interesting problems, ranging from noisy locomotion to transport of motor proteins, that establish a connection between Brownian motors and stochastic resonance.

\section{Acknowledgements}

The author gratefully acknowledges helpful discussions with Alexander Neiman, Frank Moss, Lutz Schimansky-Geier, Jan Freund, Igor Sokolov and Peter Hänggi. Financial support from UNAM through project DGAPA-IN-111000, is acknowledged. The author also wants to thank the Alexander von Humboldt Foundation for support.

\section{References}

[1] L. Gammaitoni, P. Hänggi, P. Jung, F. Marchesoni, Rev. Mod. Phys. 70 (1998) 223.

[2] R. D. Astumian, F. Moss, Chaos 8 (1998) 533.

[3] P. Hänggi, ChemPhysChem 3 (2002) 285.

[4] R. D. Astumian, P. Hänggi, Physics Today 55, No. 11 (2002) 33.

[5] P. Reimann, Phys. Rep. 361 (2002) 57.

[6] P. Reimann, P. Hänggi, Applied Physics A 75 (2002) 169.

[7] V. S. Anishchenko, V. V. Astakhov, A. B. Neiman, T. E. Vadivasova, L. Schimansky-Geier, Nonlinear Dynamics of Chaotic and Stochastic Systems, Springer-Verlag, Berlin, 2002.

[8] R. D. Vale, R. A. Milligan, Science 288 (2000) 88.

[9] D. Keller, C. Bustamante, Biophysical Journal 78 (2000) 541.

[10] J. Howard, Mechanics of Motor Proteins and the Cytoskeleton, Sinauer Associated, Inc., Sunderland, Massachusetts, 2001. 
[11] I. Derényi, T. Vicsek, Proc. Natl. Acad. Sci. USA 93 (1996) 6775.

[12] G. N. Stratopoulos, T. E. Dialynas, G. P. Tsironis, Phys. Lett. A 252 (1999) 151.

[13] S. Klumpp, A. Mielke, C. Wald, Phys. Rev. E 63 (2001) 031914.

[14] T. C. Elston, D. You, C. S. Peskin, SIAM J. Appl. Math. 61 (2000) 776.

[15] M. Bier, Phys. Rev. Lett. 91 (2003) 148104.

[16] R. Kanada, K. Sasaki, Phys. Rev. E 67 (2003) 061917.

[17] D. Dan, A. M. Jayannavar, G. I. Menon, Physica A 318 (2003) 40.

[18] H.-Y. Wang, J.-D. Bao, Physica A 337 (2004) 13.

[19] C. L. Asbury, A. N. Fehr, S. M. Block, Science 302 (2003) 2130.

[20] W. R. Shief, R. H. Clark, A. H. Crevenna, J. Howard, Proc. Natl. Acad. Sci. USA 101 (2004) 1183.

[21] A. Yildiz, M. Tomishige, R. D. Vale, P. R. Selvin, Science 303 (2004) 676.

[22] A. Yildiz, J. N. Forkey, S. A. McKinney, T. Ha, Y. E. Goldman, P. R. Selvin, Science 300 (2003) 2061.

[23] G. E. Snyder, T. Sakamoto, J. A. Hammer III, J. R. Sellers, P. R. Selvin, Biophys. J. 87 (2004) 1776.

[24] A. Yildiz, H. Park, D. Safer, Z. Yang, L.-Q. Chen, P. R. Selvin, H. L. Sweeney, J. Biol. Chem. 279 (2004) 37223.

[25] Z. Ökten, L. S. Churchman, R. S. Rock, J. A. Spudich, Nature Struct. \& Mol. Biol. 11 (2004) 884.

[26] J. L. Mateos, A. Neiman, F. Moss, in: S. M. Bezrukov (Ed.), Unsolved Problems of Noise and Fluctuations UPON 2002, AIP Conference Proceedings 665 (2003) 569 .

[27] J. L. Mateos, A. Neiman, F. Moss, J. A. Freund, L. Schimansky-Geier, I. M. Sokolov, in: L. Schimansky-Geier, D. Abbot, A. Neiman, C. Van den Broeck (Eds.), Noise in Complex Systems and Stochastic Dynamics, Proc. of SPIE 5114 (2003) 20.

[28] J. L. Mateos, Fluct. Noise Lett. 4 (2004) L161.

[29] J. L. Mateos, Phys. Rev. Lett. 84 (2000) 258.

[30] W. H. Press, S. A. Teukolsky, W. T. Vetterling, B. P. Flannery, Numerical Recipes in Fortran. The Art of Scientific Computing, Second edition, Cambridge University Press, New York, NY, 1994. 